Article

\title{
Preparation of Vitexin Nanoparticles by Combining the Antisolvent Precipitation and High Pressure Homogenization Approaches Followed by Lyophilization for Dissolution Rate Enhancement
}

\author{
Chengbo Gu ${ }^{1, *}$, Ziwei Liu ${ }^{1}$, Xiaohan Yuan ${ }^{2}$, Wang $\mathrm{Li}^{1}$, Yuangang $\mathrm{Zu}^{1}$ and Yujie Fu ${ }^{1, *}$ \\ 1 Key Laboratory of Forest Plant Ecology, Ministry of Education, Northeast Forestry University, \\ Harbin 150040, China; ziwei17liu@163.com (Z.L.); wanglisouthernsea@126.com (W.L.); \\ zuyuangang@163.com (Y.Z.) \\ 2 Life Science and Biotechnique Research Center, Northeast Agricultural University, Harbin 150030, China; \\ yuanxiaohana@163.com \\ * Correspondence: chengbo.gu@nefu.edu.cn (C.G.); yujie_fu@163.com (Y.F.); \\ Tel.: +86-451-8219-2286 (C.G.); +86-451-8219-0535 (Y.F.)
}

Received: 23 October 2017; Accepted: 18 November 2017; Published: 22 November 2017

\begin{abstract}
Vitexin, a natural flavonoid found in many medicinal plants, is well known for its rich pharmacological activities. However, the poor water solubility of vitexin has limited its therapeutic application. The aim of this study was to prepare the nanoparticles of vitexin by combining the antisolvent precipitation (ASP) and high pressure homogenization (HPH) approaches followed by lyophilization for improving the dissolution rate of this poorly water-soluble drug. The effects of main factors influencing the mean particle size (MPS) of vitexin were investigated and optimized. Under optimum conditions, vitexin nanosuspensions with an MPS of $80.5 \mathrm{~nm}$ were obtained and then lyophilized to form nanoparticles. The obtained vitexin nanoparticles were further characterized by scanning electron microscopy (SEM), Fourier transform infrared spectroscopy (FTIR), mass spectrometry (MS), X-ray powder diffraction (XRPD), gas chromatography (GC) and dissolution testing. The results showed that the nanoparticles of vitexin were converted into an amorphous form, with its chemical structure unchanged. Additionally, the residual dimethyl sulfoxide (DMSO) is lower than the International Conference on Harmonization (ICH) limit for class 3 solvents. The dissolution rate of processed vitexin was significantly higher (5.58-fold) than that of raw drug. Overall, the combinative process we developed is an effective way to produce vitexin nanoparticles with markedly enhanced dissolution rate.
\end{abstract}

Keywords: nanotechnology; nanoparticles; vitexin; dissolution rate

\section{Introduction}

Vitexin (apigenin-8-C-glucoside, as shown in Figure 1) is a naturally occurring flavone glucoside, which was found in many medicinal plant species such as Crataegus pinnatifida [1], Cajanus cajan [2], Livistona chinensis [3], Mimosa pudica [4], Phyllostachys edulis [5], and Trollius chinensis [6]. Vitexin has received increasing attention due to its various pharmacological effects, such as antihypotensive, anti-inflammatory, anticancer, antispasmodic, antimicrobial, antioxidant, antithyroid and antiarteriosclerotic effects [7,8]. It is also used for heart disease prevention [9]. 
<smiles>O=c1cc(-c2ccc(O)cc2)oc2c([C@@H]3O[C@H](CO)[C@@H](O)[C@H](O)[C@H]3O)c(O)cc(O)c12</smiles>

Figure 1. Chemical structural formula of vitexin.

Despite having multiple medicinal benefits, the low aqueous solubility and poor bioavailability of vitexin limit its clinical application $[9,10]$. To improve the water solubility of vitexin, micronized vitexin particles was made using the supercritical antisolvent technology, but it has the limitations of low yield and high equipment cost during the preparation process [9].

Nanoparticle engineering is one of the recent strategies applied to solve the problems of poorly aqueous soluble compounds [11]. Standard methods employed for the preparation of nanoparticles can be basically classified into top-down and bottom-up techniques [12]. In top-down methods, nanoparticles are produced by breaking down the large particles to smaller particle sizes by mechanical attrition such as high pressure homogenization (HPH) and wet milling [13-15]. Although the top-down approaches are useful on the commercial front, these processes suffer from certain drawbacks like high energy costs, time-consuming and broad particle size distributions [16,17]. For the bottom-up approach, nanoparticles were produced by precipitation. The antisolvent precipitation (ASP) is attractive to most of the bottom-up methods. In this process, the drug is dissolved in a water-miscible organic solvent and then precipitated by addition of an antisolvent under rapid mixing [18-20]. One of the main challenges of this process is the crystal growth after the precipitation [21].

In order to improve the particle size reduction effectiveness, a combination of two techniques (bottom-up and top-down) has also been employed, such as Nanoedge (a combination of precipitation and HPH) and Smart Crystal (H42 process: a combination of spray drying with HPH, and the H96 process: a combination of freeze drying and HPH) [22].

However, three combination technologies have been scarcely explored for preparation of nanoparticles. In the present study, we employed a combination of ASP and HPH approaches followed by lyophylization to prepare vitexin nanoparticles. Various factors affecting the mean particle size (MPS) of vitexin, including vitexin solution concentration, antisolvent to solvent volume ratio, stirring speed, precipitation temperature, homogenization pressure and cycle number, were investigated and optimized by an orthogonal array design (OAD) and single factor optimization design. The obtained vitexin nanoparticles were analyzed through scanning electron microscopy (SEM), Fourier transform infrared spectroscopy (FTIR), liquid chromatography-mass spectrometry (LC-MS), X-ray powder diffraction (XRPD), gas chromatography (GC), and dissolution testing. To the best of our knowledge, there is no study exploring the use of a combination method of ASP-HPH and lyophilization to produce vitexin nanoparticles.

\section{Results and Discussion}

\subsection{Optimization of the ASP Process}

The first step in the ASP process is to optimize the operating conditions to obtain a minimum MPS of micronized vitexin. In the present study, an orthogonal array design OAD9 $\left(3^{4}\right)$ was applied to evaluate the effects of the following factors on the MPS of vitexin particles: concentration of the vitexin solution (A), antisolvent to solvent volume ratio (B), stirring speed (C), and precipitation 
temperature (D). The assignment of the experiment and the collected data for MPS of micronized vitexin is in Table 1. The results in Table 1 indicated that the maximum MPS of vitexin particles was $1129.2 \pm 95.25 \mathrm{~nm}$, and the minimum was $368.4 \pm 31.23 \mathrm{~nm}$. It can be concluded from Table 1 that the influence to the MPS of micronized vitexin decreases in the order: $\mathrm{D}>\mathrm{A}>\mathrm{B}>\mathrm{C}$ according to the $R$-values. In addition, the minimum MPS of vitexin particles was obtained by numerical analysis using the Design-Expert 8.0.5 software (Stat-Ease Inc., Minneapolis, MN, USA) as below: when vitexin solution concentration, antisolvent-solvent volume ratio, stirring speed, and reaction temperature were A1B2C3D1 (25 mg/mL, 15 times, $1500 \mathrm{rpm}$, and $\left.4{ }^{\circ} \mathrm{C}\right)$, respectively. Through a validation test, smaller micronized vitexin was obtained with a MPS of $368.4 \pm 31.23 \mathrm{~nm}$.

Table 1. OAD9 $\left(3^{4}\right)$ and experimental results.

\begin{tabular}{cccccc}
\hline Trial No. & A & B & C & D & $\begin{array}{c}\text { MPS (nm) } \pm \text { SD } \\
(\boldsymbol{n}=\mathbf{3})\end{array}$ \\
\hline 1 & $1(25)$ & $1(5)$ & $1(500)$ & $1(4)$ & $425.8 \pm 45.22$ \\
2 & $1(25)$ & $2(15)$ & $2(1000)$ & $2(25)$ & $404.4 \pm 34.83$ \\
3 & $1(25)$ & $3(25)$ & $3(1500)$ & $3(35)$ & $775.8 \pm 29.12$ \\
4 & $2(35)$ & $1(5)$ & $2(1000)$ & $3(35)$ & $1129.2 \pm 95.25$ \\
5 & $2(35)$ & $2(15)$ & $3(1500)$ & $1(4)$ & $368.4 \pm 31.23$ \\
6 & $2(35)$ & $3(25)$ & $1(500)$ & $2(25)$ & $817.6 \pm 70.69$ \\
7 & $3(45)$ & $1(5)$ & $3(1500)$ & $2(25)$ & $880.9 \pm 50.18$ \\
8 & $3(45)$ & $2(15)$ & $1(500)$ & $3(35)$ & $1073.6 \pm 88.90$ \\
9 & $3(45)$ & $3(25)$ & $2(1000)$ & $1(4)$ & $459.0 \pm 28.92$ \\
$K_{1}{ }^{(a)}$ & $535.33 \pm 36.39$ & $901.967 \pm 65.81$ & $772.333 \pm 68.27$ & $417.733 \pm 35.12$ & \\
$K_{2}$ & $861.73 \pm 67.98$ & $615.467 \pm 51.65$ & $754.2 \pm 55.26$ & $700.967 \pm 51.90$ & \\
$K_{3}$ & $804.50 \pm 56.00$ & $684.133 \pm 42.91$ & $675.033 \pm 36.84$ & $1082.867 \pm 73.35$ & \\
$R^{(b)}$ & 326.40 & 286.50 & 97.30 & 665.13 & \\
Optimal level & A1 & B2 & C2 & D1 & \\
\hline
\end{tabular}

A: concentration of vitexin solution $(\mathrm{mg} / \mathrm{mL})$; B: antisolvent to solvent volume ratio; C: stirring speed (rpm); D: reaction temperature. ${ }^{\text {(a) }} K_{i}^{\mathrm{A}}=\Sigma$ (mean particle size at $A_{i}$ )/3, the mean values of the mean particle size for a certain factor at each level with standard deviation. ${ }^{(\mathrm{b})} R_{i}^{\mathrm{A}}=\max \left\{K_{i}^{\mathrm{A}}\right\}-\min \left\{K_{i}^{\mathrm{A}}\right\}$.

The result of ANOVA analyses was shown in Table 2, the effect of the reaction temperature on the MPS of vitexin particles was significant $(p<0.05)$ among various experimental factors, and the concentration of vitexin solution, antisolvent to solvent volume ratio and stirring speed were not significant $(p>0.05)$.

Table 2. ANOVA analysis of four factors for the ASP process.

\begin{tabular}{lccccc}
\hline \multicolumn{1}{c}{ Factor } & $\begin{array}{c}\text { Sum of } \\
\text { Squares }\end{array}$ & $\begin{array}{c}\text { Degrees of } \\
\text { Freedom }\end{array}$ & F Ratio & $\boldsymbol{F}_{\mathbf{0 . 0 5}}$ & $\begin{array}{c}\text { Type of } \\
\text { Effect }\end{array}$ \\
\hline A: Concentration of vitexin solution $(\mathrm{mg} / \mathrm{mL})$ & $182,263.31$ & 2 & 11.35 & 19.00 & \\
B: Antisolvent to solvent volume ratio & $134,248.72$ & 2 & 8.36 & 19.00 & \\
C: Stirring speed (rpm) & $16,063.47$ & 2 & 1.00 & 19.00 & \multirow{2}{*}{ significant } \\
D: Reaction temperature & $668,471.08$ & 2 & 41.61 & 19.00 & \\
Error & $16,063.47$ & 2 & & & \\
\hline
\end{tabular}

\subsection{Effect of Different Influential Factors on the MPS of Vitexin Particles in the ASP Process}

The influences of various factors on the MPS of the vitexin particles are observed in Figure 2. The MPS of vitexin particles increased with vitexin solution concentration ranging from 25 to $35 \mathrm{mg} / \mathrm{mL}$, but decreased when drug concentration increasing from 35 to $45 \mathrm{mg} / \mathrm{mL}$ (as shown in Figure 2a). The MPS of vitexin particles decreased with the antisolvent to solvent volume ratio increasing from 5 to 15 times, but increased when volume ratio varied from 15 to 25 times (as shown in Figure 2b). From Figure 2c, with the stirring speed increasing from 500 to $1500 \mathrm{rpm}$, the MPS of vitexin particles decreased significantly. Figure $2 \mathrm{~d}$ showed that the reaction temperature had a large 
influence on particle size. As the reaction temperature changed from 4 to $35^{\circ} \mathrm{C}$, vitexin particle size increased significantly.
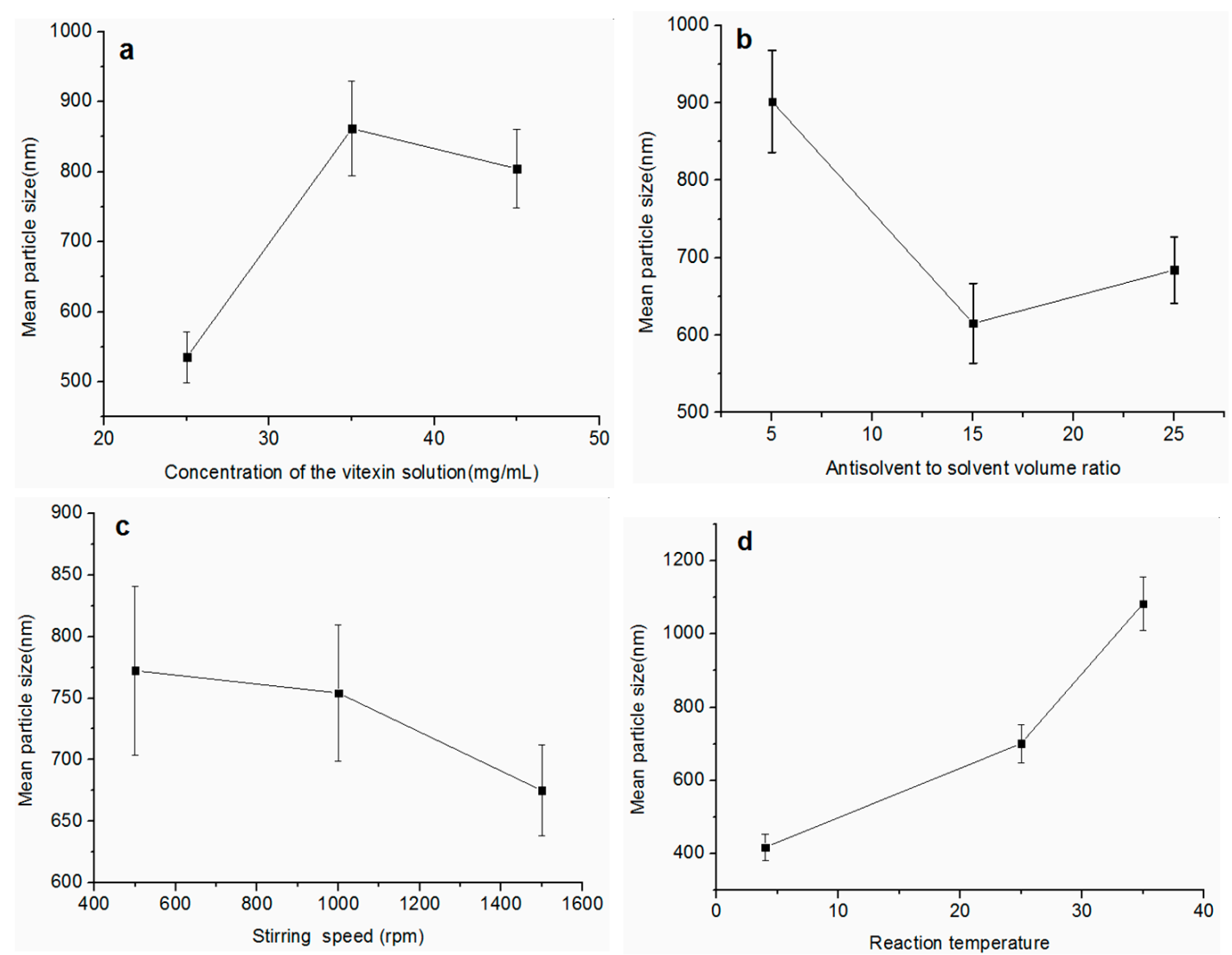

Figure 2. The effect of each factor on the MPS of vitexin particles. (a) concentration of the vitexin solution; (b) antisolvent to solvent volume ratio; (c) stirring speed; (d) reaction temperature.

\subsection{Optimization of HPH Process}

After ASP process is done, the parameters in the HPH process, such as homogenization pressure and homogenization cycles, were further optimized by single factor optimization design (shown in Figure 3). When keeping the numbers of homogenization cycle at 9, the operating conditions of homogenization pressures (200-1200 bar) were optimized, as shown in Figure 3a, increasing homogenization pressure from 200 to 1000 bar, the particle size fell. However, the higher pressure didn't lead to significant improvement in particle size when the homogenization pressure exceeded 1000 bar. Thus, the optimal homogenization pressure was considered to be 1000 bar in the present experiment.

The number of homogenization cycles is another important factor determining the fineness of the product in the HPH process. Samples were withdrawn after 5, 10, 15, 20 and 25 homogenization cycles under 1000 bar (Figure 3b). Although more cycles lead to particle size reduction, from the lower energy consumption point of view, 20 homogenization cycles were good enough to reach a homogenous nanosuspension. Therefore, the optimized HPH condition was achieved by 1000 bar for 20 cycles. One important role of the HPH step is to anneal the initial precipitates obtained by the ASP process [23].

\subsection{Characterization of Vitexin Nanoparticles}

Vitexin nanosuspensions produced under the optimal operating conditions in ASP and $\mathrm{HPH}$ processes were transformed into nanoparticles by lyophilization using $5 \%$ mannitol as the cryoprotectant, and the vitexin nanoparticles were further analyzed in terms of morphology (SEM), particle size, XRPD, LC-MS, FTIR, GC, and dissolution behavior. 

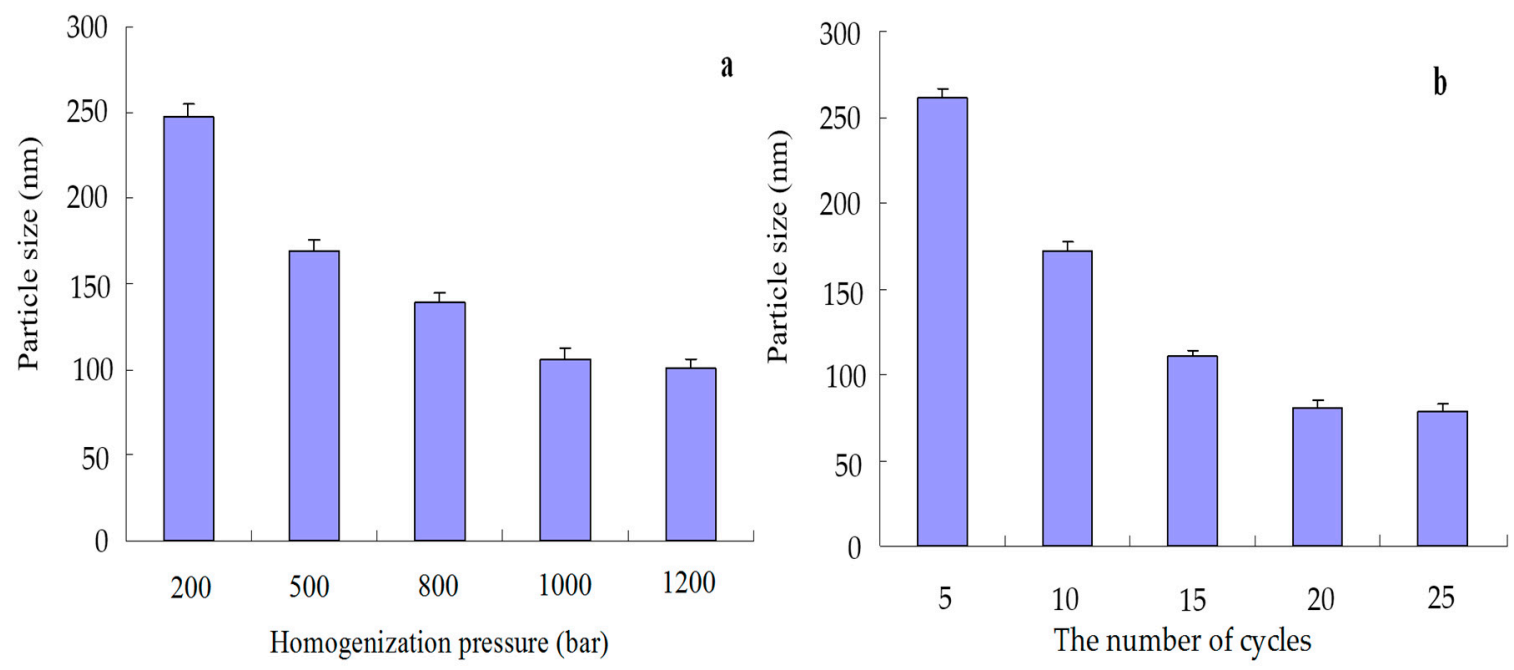

Figure 3. Effect of homogenization pressure (a) and homogenization cycles (b) on the particle size of vitexin nanosuspensions.

\subsubsection{SEM and Particle Size Analysis}

SEM images of raw and vitexin nanoparticles were shown in Figure 4. As shown in Figure 4a, the raw vitexin formed rose-like crystals with particle size distributing between 10 and $70 \mu \mathrm{m}$. However, in Figure $4 \mathrm{~b}$, vitexin nanoparticles were almost spherical with particle size ranging from 150 to $200 \mathrm{~nm}$. Vitexin nanoparticles were significantly smaller and more uniform than raw vitexin, which indicates better solubility. Figure 4c showed the particle size distribution of vitexin nanosuspensions and redissolved nanosized vitexin. The vitexin nanosuspensions with an MPS of $80.5 \mathrm{~nm}$ were obtained under optimum preparation conditions. Compared to vitexin nanosuspensions, the MPS of redissolved vitexin nanopartilces $(108 \mathrm{~nm})$ increased slightly. The major reason for this phenomenon may be explained by the agglomeration of vitexin particles during the freeze drying. Furthermore, the zeta potential values of the vitexin particles obtained through ASP and vitexin nanosuspensions with poloxamer 188 as a stabilizer by ASP-HPH were determined as -3.2 and $-18.5 \mathrm{mV}$, respectively. Poloxamers 188, a nonionic linear copolymers with surfactant properties, was often used as stabilizing, solubilizing or emulsifying agents in pharmaceutical formulations of poorly water soluble drugs [24]. In this study, as an efficient steric stabilizer, poloxamer 188 increased the physical stability of vitexin nanoparticles.

\subsubsection{FTIR Assay and LC-MS Assay}

In FT-IR spectra (Figure 5a), raw vitexin showed characteristic peaks at 3383 (hydroxyl) and $1654 \mathrm{~cm}^{-1}$ (carbonyl), as well as at 1614, 1508 and $1429 \mathrm{~cm}^{-1}$ (aromatic double bond). The presence of all major peaks of vitexin in physical mixture (Figure $5 \mathrm{~d}$ ) and freeze-dried sample (Figure 5e) ruled out any chemical interaction between vitexin and poloxamer 188. It implied that poloxamer 188 stabilizes vitexin nanoparticles just by physical interaction namely, adsorbing onto the surface of vitexin nanoparticles formed. Furthermore, the chemical structure of vitexin was not changed before and after the formulation and not influenced by excipients in the physical mixture.

The mass spectrograms of raw vitexin and vitexin nanoparticles are shown in Figure 6, in the chart, the molecular weight of unprocessed vitexin and vitexin nanoparticles were both 431.4. Combining the results of FTIR and MS, the molecular structure of nanosized vitexin did not change in the preparation process. 

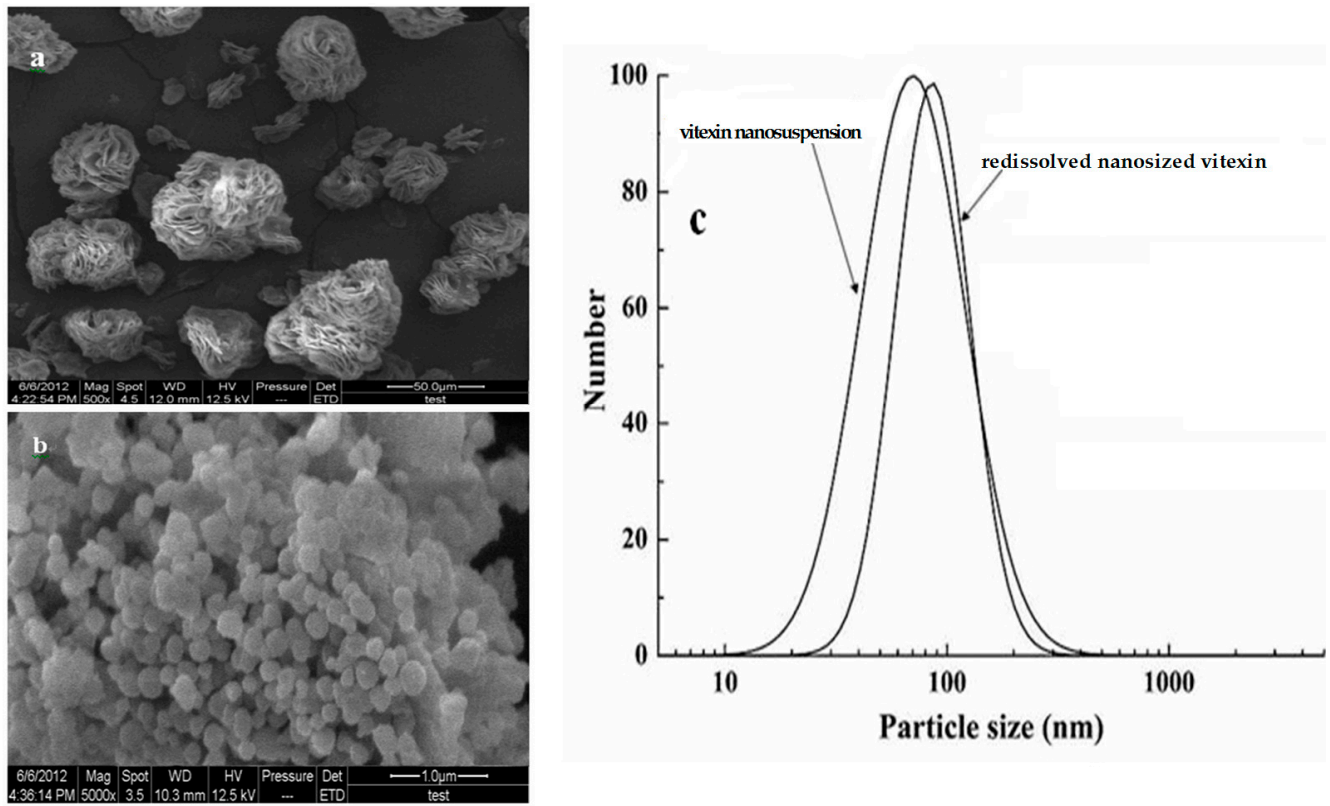

Figure 4. SEM images of (a) raw vitexin, (b) vitexin nanoparticles, and (c) Particle size distribution (PSD) of redissolved vitexin nanoparticles.

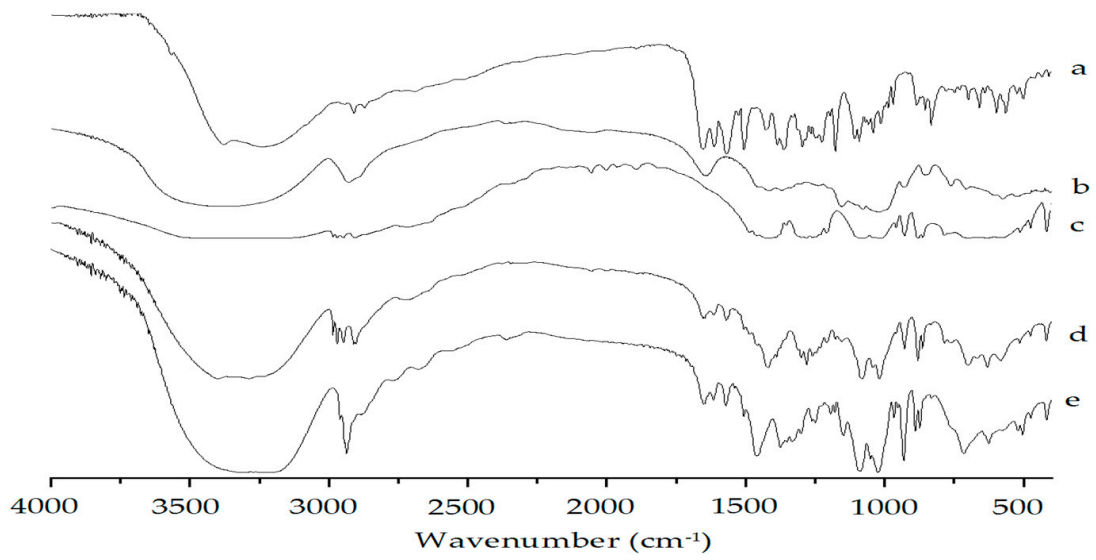

Figure 5. FTIR spectra of (a) raw vitexin; (b) poloxamer 188; (c) mannitol; (d) physical mixture of vitexin; poloxamer 188 and mannitol; and (e) vitexin nanoparticles.

\subsubsection{XRD Analysis}

The X-ray diffraction results for raw vitexin, blank excipients, physical mixture and vitexin nanoparticles are shown in Figure 7. As shown in Figure 7a, diffraction peaks of raw vitexin were observed at the diffraction angles of $2 \theta=7.66^{\circ}, 10.40^{\circ}, 11.24^{\circ}, 14.12^{\circ}, 15.36^{\circ}, 16.6^{\circ}, 17.8^{\circ}, 18.72^{\circ}, 20.56^{\circ}$, $23.20^{\circ}, 25.04^{\circ}, 26.80^{\circ}$ and $29.10^{\circ}$. In Figure $7 \mathrm{~d}$, characteristic diffraction peaks of physical mixture were almost the same as those of mannitol. Diffraction peaks of raw vitexin might be covered or overlapped by those of mannitol. The vibration intensity of the diffraction peaks of the vitexin nanoparticles obviously decreased compared with physical mixture, and the diffraction peaks of mannitol can be observed in physical mixture or vitexin might be present in an amorphous state. Changes in crystallinity of mannitol could occur during the freeze-drying process, which caused its characteristic diffraction peaks to become weak or disappear. Vitexin nanoparticles were found a complete lack of any diffraction peak compared to raw vitexin, which implied that the nanosized vitexin existed in the amorphous state instead of crystalline form. It was reported that the lyophilization process step can modify the structure of drug powder $[25,26]$. 
-Q1: 10 MCA scans from Sample 1 (TuneSampleID) of yuanyao q1-...

Max. 1.8e7 cps.

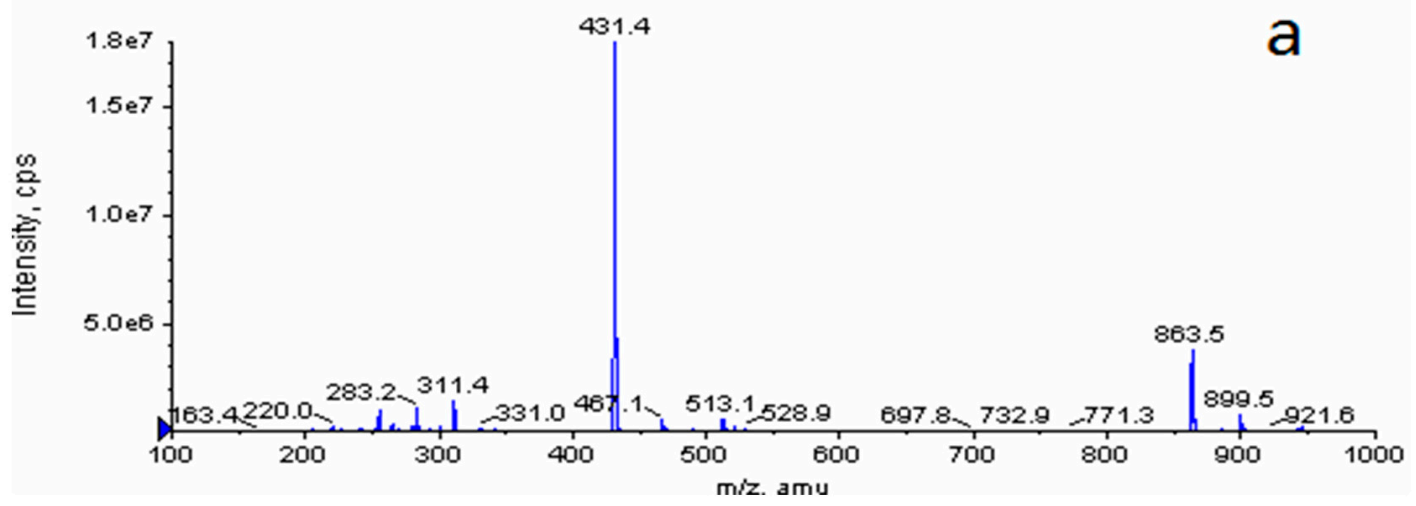

-Q1: 10 MCA scans from Sample 1 (TuneSampleID) of namiyao q1-..

Max. $4.0 \mathrm{e} 6 \mathrm{cps}$.

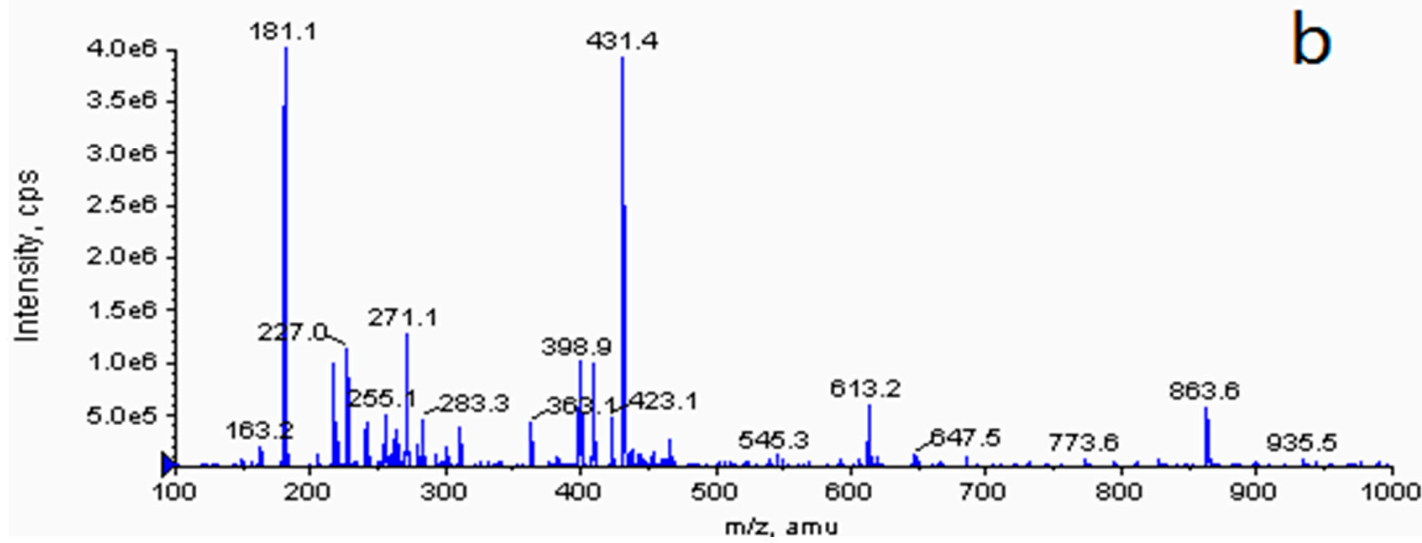

Figure 6. LC-MS spectra of (a) raw vitexin and (b) vitexin nanoparticles.

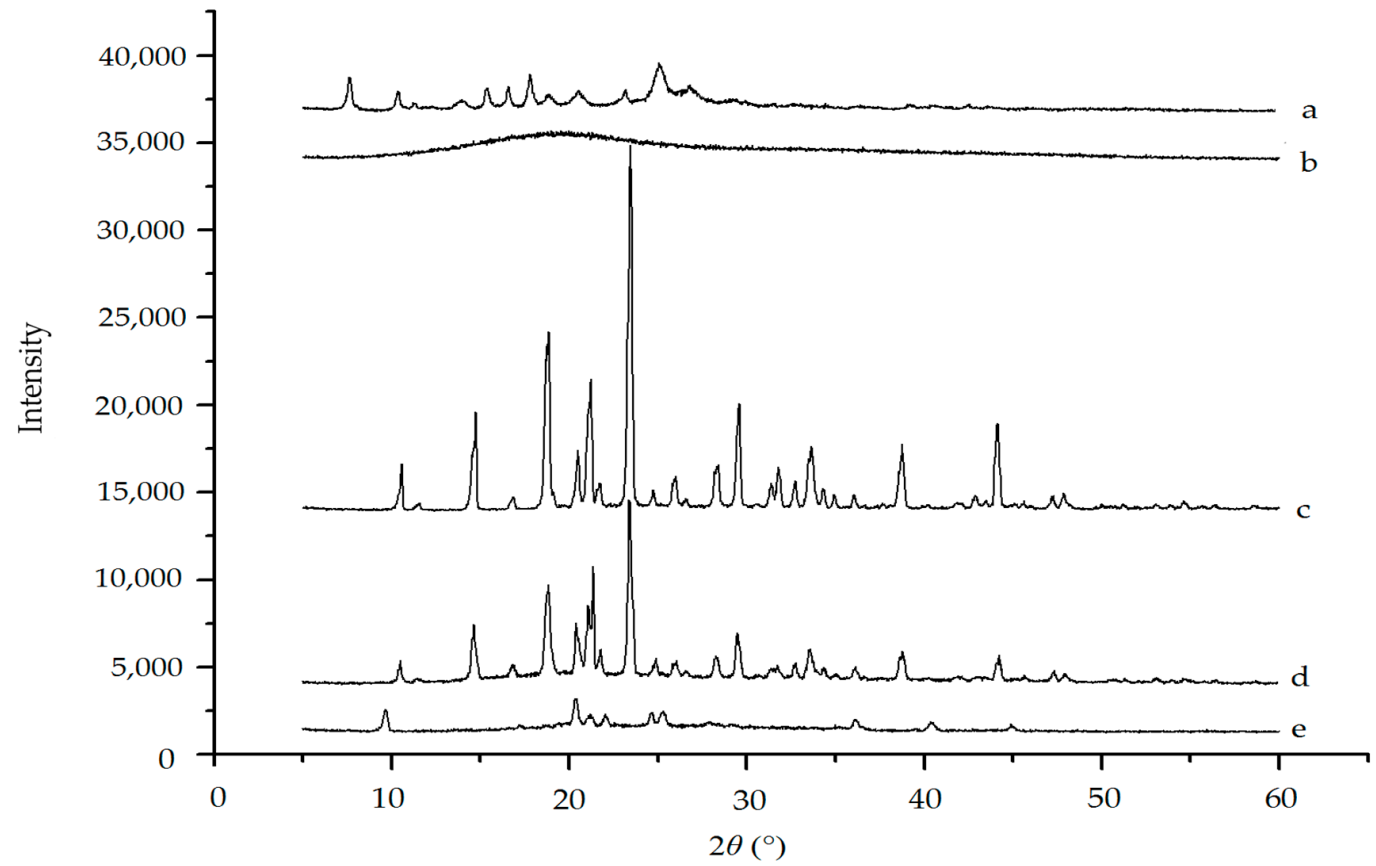

Figure 7. XRPD spectrum of (a) raw vitexin; (b) poloxamer 188; (c) mannitol; (d) physical mixture; and (e) vitexin nanoparticles. 


\subsubsection{Residual Solvent Determination}

Dimethyl sulfoxide (DMSO), a class 3 solvent with low toxicity in humans, was applied to prepare vitexin particles through the ASP process. Figure 8 showed the GC analysis results of residual DMSO. Seen from Figure $8 \mathrm{a}, \mathrm{H}_{2} \mathrm{O}$ and DMSO were eluted at 1.2 and $6.6 \mathrm{~min}$, respectively. A linear regression equation of peak area $(Y)$ versus DMSO concentration $(x)$ was obtained as $Y=0.7235 x-25.608$ $\left(R^{2}=0.9988\right)$ over the range of $0.2-5 \mathrm{mg} / \mathrm{mL}$. Based on the equation, the amount of residual DMSO in vitexin particles is 1757 ppm, which is much lower than the ICH limit (below 5000 ppm) and is acceptable for pharmaceutical use.
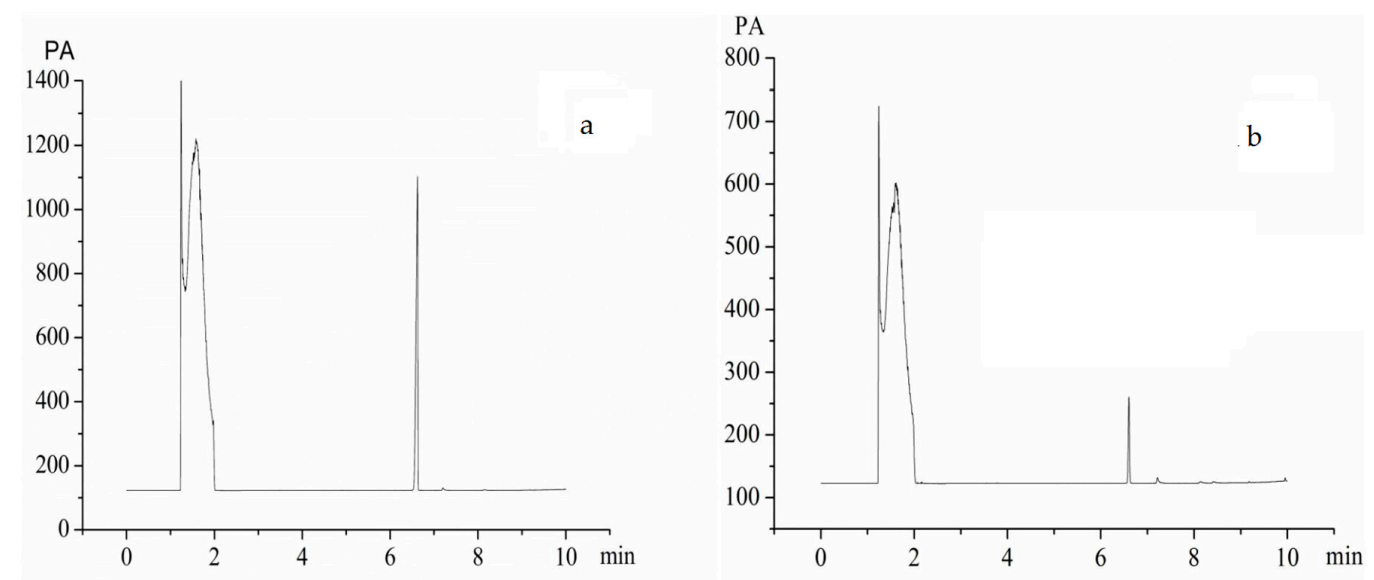

Figure 8. Gas chromatograms of samples (a) DMSO standard solution; and (b) vitexin processed by the ASP process.

\subsubsection{Dissolution Rate Analysis}

As seen in Figure 9, obvious differences were observed between the two in dissolution curves. Vitexin nanoparticles showed improved dissolution rate as compared to raw vitexin. In the $5 \mathrm{~min}$, the in vitro dissolution rates of raw vitexin and nanoparticles were around $16.85 \%$ and $93.94 \%$, respectively. However, at $30 \mathrm{~min}$, dissolution rates of raw vitexin and nanoparticles were found to be around $31.17 \%$ and $96.58 \%$, respectively. The accelerated dissolution rate of vitexin nanoparticles could be mainly explained by the particle size reduction, resulting in a significant increase in specific surface area $[27,28]$.

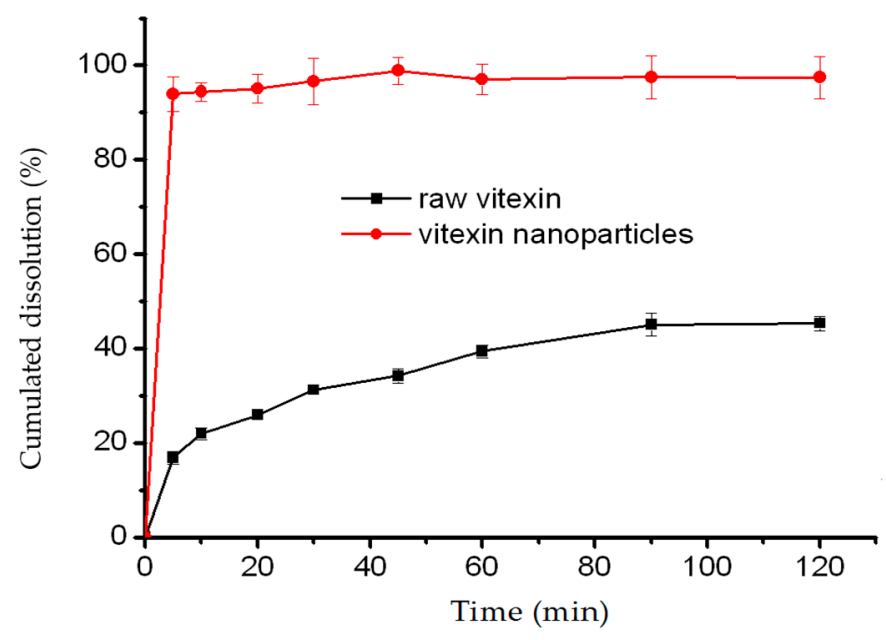

Figure 9. Dissolution profiles of raw vitexin and vitexin nanoparticles. 


\section{Materials and Methods}

\subsection{Materials}

Vitexin (FW = 432.4, purity 99.7\%) was provided from Chengdu Biopurify Phytochemicals Ltd. (Chengdu, China). Dimethyl sulfoxide (DMSO, purity $\geq 98.5 \%$ ) and acetonitrile of chromatographic grade were purchased from Sigma Aldrich (Steinheim, Germany). Poloxamer 188 was purchased from German BASF Company (Ludwigshafen, Germany). Deionized water was purified by a Milli-Q water purification system (Millipore Co., Bedford, MA, USA).

\subsection{Preparation of Vitexin Nanosuspensions by the ASP-HPH Approach}

\subsubsection{ASP Process}

In preliminary experiments, the solubility of raw vitexin in different organic solvents such as DMSO, ethanol, acetone, isopropanol, n-butanol, ethyl acetate and $N$-methylpyrrolidone was investigated, respectively. It was found that vitexin shows the good solubility only in DMSO. Hence, DMSO was selected as a solvent in the ASP process. The ASP process is conducted as follows: DMSO and deionized water were used as solvent and antisolvent, respectively. A certain amount of raw vitexin was completely dissolved in DMSO at different concentrations. The obtained drug solution was then poured into deionized water with vigorous magnetic stirring. Vitexin particles immediately precipitated from the solution upon mixing. The stirring intensity and temperature were controlled by a temperature-controlled magnetic stirrer. After stirring for $6 \mathrm{~min}$, micro/nanoparticle suspensions were centrifuged at 12,000 rpm for $10 \mathrm{~min}$ and washed three times with deionized water.

The factors affecting particle size such as vitexin solution, antisolvent to solvent volume ratio, stirring speed and precipitation temperature were optimized by an orthogonal array design OAD9 $\left(3^{4}\right)$, which was shown in Table 3. The optimization of the ASP process was carried out with four factors and three levels, namely, concentration of vitexin solution $(25,35,45 \mathrm{mg} / \mathrm{mL})$, antisolvent to solvent volume ratio $(5,15,25)$, stirring speed $(500,1000,1500 \mathrm{rpm})$ and precipitation temperature $\left(4,25,35^{\circ} \mathrm{C}\right)$. According to the preliminary experimental results, the ranges and levels of influencing factors were confirmed. The MPS (nm) of vitexin particles was the dependent variable. The data were analyzed using the Design-Expert software (Version 8.0.5, Stat-Ease Inc., Minneapolis, MN, USA). Differences at a level of $p<0.05$ were considered statistically significant.

Table 3. Factors and levels of the OAD.

\begin{tabular}{ccccc}
\hline \multirow{2}{*}{ Factor/Level } & A & B & C & D \\
\cline { 2 - 5 } & $\begin{array}{c}\text { Concentration of Vitexin } \\
\text { Solution (mg/mL) }\end{array}$ & $\begin{array}{c}\text { Antisolvent to } \\
\text { Solvent Volume Ratio }\end{array}$ & $\begin{array}{c}\text { Stirring Speed } \\
\text { (rpm) }\end{array}$ & $\begin{array}{c}\text { Precipitation } \\
\text { Temperature }\left({ }^{\circ} \mathbf{C}\right)\end{array}$ \\
\hline 1 & 25 & 5 & 500 & 4 \\
2 & 35 & 15 & 1000 & 25 \\
3 & 45 & 25 & 1500 & 35 \\
\hline
\end{tabular}

\subsubsection{HPH Process}

Subsequently, $50 \mathrm{mg}$ of vitexin particles obtained under the optimal ASP conditions were dissolved in $50 \mathrm{~mL}$ distilled water containing 5\% $(v / w)$ Poloxamer 188 as stabilizers to prevent the aggregation of particles in suspensions.

The coarse suspensions were homogenized by a high pressure homogenizer (AH100D, ATS Engineering Inc., Vancouver, BC, Canada) under different operating conditions (homogenization pressure varying from 200 to 1200 bars and cycle numbers ranging from 5 to 25). The effects of homogenization pressure and cycle number on the MPS of vitexin nanosuspensions were optimized by a single factor optimization design. The particle size was determined by particle size analyzer laser (ZetaPALS, Brookhaven Instruments Co., Holtsville, NY, USA). 


\subsubsection{Lyophilization}

The obtained vitexin nanosuspensions were then lyophilized by the addition of $5 \%(w / v)$ mannitol as a cryoprotectant. The nanosuspensions were prefrozen in the refrigerator (DW-86L, Haier, Qingdao, China) at $-40{ }^{\circ} \mathrm{C}$ for $24 \mathrm{~h}$ and then lyophilized using a lyophilizer (Beijing Boyikang Lab Instrument Co., Ltd., Beijing, China) at $-50{ }^{\circ} \mathrm{C}$ for $48 \mathrm{~h}$. Freeze-dried samples were used for solid state characterization.

\subsection{Characterization Vitexin Nanoparticles}

\subsubsection{Morphology, MPS and Zeta Potential Measurement}

The surface morphology of raw vitexin and vitexin nanoparticles were observed by SEM (Quanta 200, FEI Company, Eindhoven, The Netherlands). The MPS of vitexin nanosuspensions and the particle size distribution (PSD) of lyophilized powder dispersed in deionized water were measured with dynamic light scattering (DLS) (Zeta-PALS, Brookhaven Instruments Corp., Holtsville, NY, USA). The zeta potential values of vitexin particles obtained through ASP and vitexin nanosuspensions prepared by ASP-HPH techniques were measured using a PALS Zeta Potential Analyzer (Brookhaven Instruments Corp., Holtsville, NY, USA) with the samples diluted in deionized water to approximately $1 \mathrm{mg} / \mathrm{mL}$ for each measurement. All measurements were made in triplicate.

\subsubsection{FTIR Analysis}

Chemical bond status of the samples was analyzed with FTIR (IRAFFINITY-1, Shimadzu Co. Kyoto, Japan.) spectra using $\mathrm{KBr}$ in the wave number range of $4000-500 \mathrm{~cm}^{-1}$ at a resolution of $4 \mathrm{~cm}^{-1}$.

\subsubsection{LC-MS Analysis}

The raw vitexin and vitexin nanoparticles were dissolved separately in HPLC grade methanol. An Agilent 1100 series HPLC system (Agilent Inc., Palo Alto, CA, USA) consisted of a G1312A binary pump, a 7725i manual injector, and a G1379A degasser. The analytical column employed was a HiQ sil C18W $(4.6 \mathrm{~mm} \phi \times 250 \mathrm{~mm}, 5 \mu \mathrm{m})$. The mobile phase was methanol-water-formic acid (15:84.99:0.01, $v / v / v)$. The flow rate was $1 \mathrm{~mL} / \mathrm{min}$ and the column temperature was $25^{\circ} \mathrm{C}$. The column effluent was monitored by an API3000 triple quadrupole mass spectrometer (Biosystems, Toronto, ON, Canada) with an electrospray ionization (ESI) source, which was connected to the liquid chromatography system. Ionization of vitexin was obtained in the negative ion mode. Compound parameters, viz. declustering potential (DP) and entrance potential (EP) were set at -70 and $-10 \mathrm{~V}$, respectively, for analysis of vitexin. The Analyst 1.4 of API3000 (AB, Foster City, CA, USA) was used for data acquisition.

\subsubsection{XRPD Analysis}

XRPD analysis of different samples was measured by X-ray diffraction (JDX-3530, JEOL, Tokyo, Japan). The pattern was scanned in the range of diffraction angle $2 \theta=5-60^{\circ}$ at a rate of $0.02^{\circ} / \mathrm{min}$ with a step length of $2 \theta=0.02^{\circ}$ using $\mathrm{Cu} \mathrm{K} \alpha 1$ radiation at $40 \mathrm{kV}$ and $40 \mathrm{~mA}$.

\subsubsection{Residual DMSO Determination}

GC analysis of the residual DMSO in ASP process was determined by an Agilent $6890 \mathrm{~N}$ gas chromatograph (Agilent Technologies, Palo Alto, CA, USA) equipped with a flame ionization detector (FID) detector and a HP- 5 capillary column $(30 \mathrm{~m} \times 0.32 \mathrm{~mm} \times 0.25 \mu \mathrm{m}, 5 \%$ phenyl methyl siloxane). In addition, $2.0 \mathrm{~g}$ of vitexin particles was dissolved in $10 \mathrm{~mL}$ deionized water. The vitexin solution was shaken by ultrasonic irradiation at $25^{\circ} \mathrm{C}$ for $60 \mathrm{~min}$. The sample was then centrifuged at $15,000 \mathrm{rpm}$ for $15 \mathrm{~min}$, and $5.0 \mu \mathrm{L}$ of resulting supernatants was injected into the GC injection port. The GC settings were programmed as follows: the initial oven temperature was maintained at $100{ }^{\circ} \mathrm{C}$ for 
$3 \mathrm{~min}$, and ramped at $2{ }^{\circ} \mathrm{C} / \mathrm{min}$ to $200{ }^{\circ} \mathrm{C}$. Nitrogen was employed as a carrier gas with a flow rate of $1 \mathrm{~mL} / \mathrm{min}$. Both the inlet and the detector temperatures were kept at $250{ }^{\circ} \mathrm{C}$ and the GC was run in a split mode set at a 1:1. Each determination was done in triplicate.

\subsubsection{Dissolution Test}

The dissolution study of vitexin nanoparticles, equivalent to $20 \mathrm{mg}$ of raw vitexin, was performed by the paddle method. In addition, $200 \mathrm{~mL}$ of phosphate buffer solution $\mathrm{pH} 7.4$ at $37.0 \pm 0.5^{\circ} \mathrm{C}$ was adopted as a dissolution medium at a paddle speed of $100 \mathrm{rpm}$. The raw drug and nanoparticles were dispersed in $200 \mathrm{~mL}$ dissolution medium accordingly. Furthermore, $1 \mathrm{~mL}$ of samples was withdrawn from the dissolution vessel at 5, 10, 20, 30, 45, 60, 90 and $120 \mathrm{~min}$, filtered through a $0.22 \mu \mathrm{m}$ filters and analyzed by the previously described HPLC method. Each sample was done in triplicate. The cumulative release amount of drug versus time profiles was plotted.

\section{Conclusions}

In summary, we prepared the nanoparticles of vitexin by combining the antisolvent precipitation and high pressure homogenization approaches followed by lyophilization. The vitexin nanosuspensions with an MPS of $80.5 \mathrm{~nm}$ were firstly produced under the following optimum ASP and HPH operating conditions (i.e., vitexin solution concentration $25 \mathrm{mg} / \mathrm{mL}$, antisolvent to solvent volume ratio 15 times, stirring speed $1500 \mathrm{rpm}$, precipitation temperature $4{ }^{\circ} \mathrm{C}$, homogenization pressure 800 bar and number of homogenization cycles 20) and then lyophilized to form nanoparticles. The obtained nanoparticles of vitexin existed in an amorphous form, with its chemical structure unchanged. Additionally, the DMSO residual is much lower than the ICH limit for class 3 solvents. The dissolution rate of vitexin nanoparticles was greatly improved compared to the raw drug. Overall, the combinative process we developed is an effective way to produce vitexin nanoparticles with a markedly enhanced dissolution rate.

Acknowledgments: The authors are grateful for the financial support from the Fundamental Research Funds for the Central Universities (2572014EA02-3), the Natural Science Foundation of Heilongiiang Province of China (C2017004, C2017021), the National Key Research and Development Program of China (2016YFD0600805), and the Scientific Research Fund of Heilongjiang Provincial Education Department (12541051).

Author Contributions: Chengbo Gu and Yuangang $\mathrm{Zu}$ conceived and designed the experiments. Ziwei Liu and Wang Li performed the experiments. Xiaohan Yuan and Yujie Fu analyzed the data. Chengbo Gu and Yujie Fu wrote the manuscript. All authors read and approved the final manuscript.

Conflicts of Interest: The authors declare no conflict of interest.

$\begin{array}{ll}\text { Abbreviations } \\ \text { ASP } & \text { antisolvent precipitation } \\ \text { HPH } & \text { high pressure homogenization } \\ \text { OAD } & \text { orthogonal array design } \\ \text { MPS } & \text { mean particle size } \\ \text { ICH } & \text { International Conference on Harmonization } \\ \text { DMSO } & \text { Dimethyl sulfoxide } \\ \text { SEM } & \text { Scanning electron microscopy } \\ \text { FTIR } & \text { Fourier transform infrared spectroscopy } \\ \text { LC-MS } & \text { Liquid chromatography-mass spectrometry } \\ \text { XRPD } & \text { X-ray powder diffraction } \\ \text { GC } & \text { Gas chromatography }\end{array}$




\section{References}

1. Pan, H.F.; Wang, X.; Du, Y.L.; Li, Y.R.; Zhao, S.N.; Pan, W.D. A simple and sensitive method for the simultaneous determination of six constituents in hawthorn leaves. Anal. Methods 2014, 6, 7475-7483. [CrossRef]

2. Fu, Y.J.; Zu, Y.G.; Liu, W.; Zhang, L.; Tong, M.H.; Efferth, T.; Kong, Y.; Hou, C.L.; Chen, L.Y. Determination of vitexin and isovitexin in pigeonpea using ultrasonic extraction followed by LC-MS. J. Sep. Sci. 2008, 31, 268-275. [CrossRef] [PubMed]

3. Yao, H.; Chen, Y.; Shi, P.Y.; Hu, J.; Li, S.G.; Huang, L.Y.; Lin, J.H.; Lin, X.H. Screening and quantitative analysis of antioxidants in the fruits of Livistona chinensis R. Br using HPLC-DAD-ESI/MS coupled with pre-column DPPH assay. Food Chem. 2012, 135, 2802-2807. [CrossRef] [PubMed]

4. Zhang, J.; Yuan, K.; Zhou, W.L.; Zhou, J.A.; Yang, P. Studies on the active components and antioxidant activities of the extracts of Mimosa pudica Linn. from southern China. Pharmacogn. Mag. 2011, 7, 35-39. [PubMed]

5. Zhang, C.J.; Meng, Z.F.; Guo, X.F.; Yue, Y.D.; Tang, F.; Yu, J.; Chen, C. Simultaneous Determination of Four Flavone C-Glycosides in Phyllostachys edulis Leaves by High-Performance Liquid Chromatography with Ultraviolet Spectrometry. Spectrosc. Spect. Anal. 2014, 34, 2568-2572.

6. An, F.; Yang, G.D.; Tian, J.M.; Wang, S.H. Antioxidant effects of the orientin and vitexin in Trollius chinensis Bunge in D-galactose-aged mice. Neural Regen. Res. 2012, 7, 2565-2575. [PubMed]

7. Prabhakar, M.C.; Bano, H.; Kumar, I.; Shamsi, M.A.; Khan, M.S.Y. Pharmacological Investigations on Vitexin. Planta Med. 1981, 43, 396-403. [CrossRef] [PubMed]

8. He, M.; Min, J.W.; Kong, W.L.; He, X.H.; Li, J.X.; Peng, B.W. A review on the pharmacological effects of vitexin and isovitexin. Fitoterapia 2016, 115, 74-85. [CrossRef] [PubMed]

9. Zu, Y.; Zhang, Q.; Zhao, X.; Wang, D.; Li, W.; Sui, X.; Zhang, Y.; Jiang, S.G.; Wang, Q.; Gu, C. Preparation and characterization of vitexin powder micronized by a supercritical antisolvent (SAS) process. Powder Technol. 2012, 228, 47-55. [CrossRef]

10. Lai, F.; Pini, E.; Corrias, F.; Perricci, J.; Manconi, M.; Fadda, A.M.; Sinico, C. Formulation strategy and evaluation of nanocrystal piroxicam orally disintegrating tablets manufacturing by freeze-drying. Int. J. Pharm. 2014, 467, 27-33. [CrossRef] [PubMed]

11. Das, S.; Suresh, P.K. Nanosuspension: A new vehicle for the improvement of the delivery of drugs to the ocular surface. Application to amphotericin B. Nanomed. Nanotechnol. 2011, 7, 242-247. [CrossRef] [PubMed]

12. Keck, C.M.; Muller, R.H. Drug nanocrystals of poorly soluble drugs produced by high pressure homogenisation. Eur. J. Pharm. Biopharm. 2006, 62, 3-16. [CrossRef] [PubMed]

13. Müller, R.H.; Peters, K. Nanosuspensions for the formulation of poorly soluble drugs: I. Preparation by a size-reduction technique. Int. J. Pharm. 1998, 160, 229-237. [CrossRef]

14. Patravale, V.B.; Date, A.A.; Kulkarni, R.M. Nanosuspensions: A promising drug delivery strategy. J. Pharm. Pharmacol. 2004, 56, 827-840. [CrossRef] [PubMed]

15. Pang, J.M.; Dong, W.J.; Li, Y.H.; Xia, X.J.; Liu, Z.H.; Hao, H.Z.; Jiang, L.M.; Liu, Y.L. Purification of Houttuynia cordata Thunb. Essential Oil Using Macroporous Resin Followed by Microemulsion Encapsulation to Improve Its Safety and Antiviral Activity. Molecules 2017, 22, 293. [CrossRef] [PubMed]

16. He, Y.; Huang, Y.B.; Cheng, Y. Structure Evolution of Curcumin Nanoprecipitation from a Micromixer. Cryst. Growth Des. 2010, 10, 1021-1024. [CrossRef]

17. Krause, K.P.; Kayser, O.; Mader, K.; Gust, R.; Muller, R.H. Heavy metal contamination of nanosuspensions produced by high-pressure homogenisation. Int. J. Pharm. 2000, 196, 169-172. [CrossRef]

18. Vaculikova, E.; Cernikova, A.; Placha, D.; Pisarcik, M.; Peikertova, P.; Dedkova, K.; Devinsky, F.; Jampilek, J. Preparation of Hydrochlorothiazide Nanoparticles for Solubility Enhancement. Molecules 2016, 21, 1005. [CrossRef] [PubMed]

19. Thorat, A.A.; Dalvi, S.V. Particle formation pathways and polymorphism of curcumin induced by ultrasound and additives during liquid antisolvent precipitation. Crystengcomm 2014, 16, 11102-11114. [CrossRef]

20. Yadav, D.; Kumar, N. Nanonization of curcumin by antisolvent precipitation: Process development, characterization, freeze drying and stability performance. Int. J. Pharm. 2014, 477, 564-577. [CrossRef] [PubMed] 
21. Sinha, B.; Muller, R.H.; Moschwitzer, J.P. Bottom-up approaches for preparing drug nanocrystals: Formulations and factors affecting particle size. Int. J. Pharm. 2013, 453, 126-141. [CrossRef] [PubMed]

22. Mueller, R.H.; Keck, C.M. Second generation of drug nanocrystals for delivery of poorly soluble drugs: SmartCrystal technology. Eur. J. Pharm. Sci. 2008, 34, S20-S21. [CrossRef]

23. Shegokar, R.; Muller, R.H. Nanocrystals: Industrially feasible multifunctional formulation technology for poorly soluble actives. Int. J. Pharm. 2010, 399, 129-139. [CrossRef] [PubMed]

24. Kalvakuntla, S.; Deshpande, M.; Attari, Z.; Kunnatur, B.K. Preparation and Characterization of Nanosuspension of Aprepitant by H96 Process. Adv. Pharm. Bull. 2016, 6, 83-90. [CrossRef] [PubMed]

25. Cavatur, R.K.; Suryanarayanan, R. Characterization of phase transitions during freeze-drying by in situ X-ray powder diffractometry. Pharm. Dev. Technol. 1998, 3, 579-586. [CrossRef] [PubMed]

26. Möschwitzer, J.; Lemke, A. Method for Carefully Producing Ultrafine Particle Suspensions and Ultrafine Particles and Use Thereof. WO/2006/108637, 19 October 2006.

27. Noyes, A.A.; Whitney, W.R. The rate of solution of solid substances in their own solutions. J. Am. Chem. Soc. 1897, 19, 930-934. [CrossRef]

28. Kesisoglou, F.; Panmai, S.; Wu, Y.H. Nanosizing-Oral formulation development and biopharmaceutical evaluation. Adv. Drug Deliv. Rev. 2007, 59, 631-644. [CrossRef] [PubMed]

Sample Availability: Not available. 